\title{
The Physiological Homeostasis of Human Populations in Variable Environments
}

\author{
Lioudmila K. Goudkova \\ Moscow State University Research Institute and Museum of Anthropology, Moscow, Russia
}

\begin{abstract}
A close evolutionary relationship of physiology and ecology of organisms determines the dynamic dependence of the population physiological status in man on ecological factors. For the explanation of the stability and variability of population physiological status the concept of physiological homeostasis was applied. The investigation of physiological status in several populations of Middle Asia, Kazakhstan, North-East Asia and Khakassia has shown that reversible changes in the environment may temporarily destabilize the equilibrium in the "population-environment" system and prolonged stresses may cause a state of disadaptation. For estimation of population physiological homeostasis dependent on the environment, generalized dispersion, correlation and factor analyses are very informative. They not only mark the violation of population homeostasis, but also indicate changes in the environment. J Physiol Anthropol Appl Human Sci 24(4): 301-306, 2005 http://www.jstage.jst.go.jp/browse/jpa [DOI: 10.2114/jpa.24.301]
\end{abstract}

Keywords: physiological homeostasis, population, environment, stability, variability, adaptation, disadaptation, ecological factors

\section{Introduction}

The plentiful information on the variability of different physiological characters in different human populations was in need of a general point of view to explain the differences between them. But the traditional explanations for physiological anthropology could not solve the problem of interpreting the results. In the present work the author has applied the concept of physiological homeostasis for the explanation of the stability and variability of the physiological status of populations. The dependence of physiological homeostasis on the ecological factors is looked upon as the main mechanism, which determines the physiological structure of populations living in different environments and at different stages of the adaptation process.

The concept of physiological homeostasis (Cannon, 1932) became the theoretical basis of a new branch of physiological anthropology-human population physiology (Goudkova,
2005).

Human population physiology investigates the physiological structure of populations in concrete environments. In the evolution process the physiological mechanisms are developed as an answer to the demands of the environment and must be in accordance with those ecological factors in which the organism is living. The active existence of organisms in a variety of situations is determined by homeostasis, which can equalize the environmental temporal and space variability (Pianka, 1981). All types of adaptation are based on the homeostasis mechanism (Gorizontov, 1981). The violation of homeostasis by environmental factors or a signal of the probability of a violation through the highest regulation levels activates systems which are responsible for the adaptation. In human population physiology homeostasis, adaptation and ecological factors are looked upon from the population level as a complex of causes and effects.

The methodological basis of this branch is the systemic and integral approach. From the point of view of the systemic approach, physiological homeostasis is determined as the ability of the population to preserve the dynamic stability of its physiological status in a changing environment and the physiological status of a population is determined as a totality of physiological variables, which are interdependent on the organism level and are correlated on the population level. If we take into account these definitions the physiological structure of a population may be looked upon as an integral formation, which is ensured by an aggregate of stable connections between the separate parts of the common system.

The combination of physiological variables may be different and depends on the aim of the work. The variables in this work - the physiological blood characters - characterize separate metabolic peculiarities of the organism and are engaged in different processes of energy balance. The physiological blood levels are quantitative phenotypic characters which can change in the limits of the reaction norm. These characters belong to "rigid" constants for which even small changes can be signals for mobilizing homeostatic regulation systems, but their reactivity makes adaptations (Shmalgauzen, 1968) and the degree of the adaptation of a character may be judged relatively only to other states of this character (Solbrig and Solbrig, 1982). An original model that 
can cast light on the peculiarities of the metabolic processes not seen in usual conditions is the situation of extreme influence, which gives the same results in every biological population and causes the rise of the phenotypic variability based on a genetic-physiological complex of adaptation mechanisms (Sapunov, 1990). The aim of the present work is to analyse the variability of separate physiological characters and physiological status as a whole.

\section{Materials and Methods}

The need for comparing very different populations from the ecological point of view determined the choice of the following regions: the temperate zone (Khakassia, Kamchatka), the arctic zone (Chukot), the arid zone (Turkmenia, Karakalpakia, Kazakhstan) and a medium altitude (Kirghizia, Tajikistan). The climatic conditions of the arctic and arid zones are extreme environments. But the climate of the arid zone is considered less extreme than the arctic. The arid climate of Middle Asia is divided into two periods (seasons): from the middle of May till the middle of October it is hot and dry; the rest of the year is damp and cold.

Aboriginal residents of the above-mentioned regions, men and women from 20 to 50 years old, were investigated. The sample size was 50-150 individuals. Because of the climatic peculiarities of the Middle Asia deserts, the Turkmen and Karakalpaks were studied twice-once before the hot season and again in the hot period.

The levels of physiological blood characters-hemoglobin, serum proteins (total protein, albumen, alpha-1-, alpha-2-, beta-, gamma-globulins), serum cholesterol and glucosewere determined by standard spectrophotometer methods. The high variability of physiological blood levels demands strict unification of laboratory and fieldwork. In Fig. 1 are given the results of canonical analysis of twenty samples. In eighteen of them the physiological blood levels were determined by comparable methods and in two by a different method. It is quite clear that such placing of groups is artificial and represents differences in methods. In our case all physiological blood levels were determined by the author and it is very important for correctly calculating the estimates of intragroup and intergroup variability of the physiological status of populations. The estimation of adaptability was done with the approach of systemic analysis, which includes different methods of univariate and multivariate biometrics.

\section{Results and Discussion}

For the purpose of classifying populations by their physiological status, cluster analysis was carried out (Fig. 2). Khakass, Kamchadals and recent migrants to Kamchatka formed one cluster. In spite of a general similarity, the physiological status of a separate group can have peculiarities. For example, the Kamchatka migrants have relatively low gamma-globulin levels, which is connected with strained

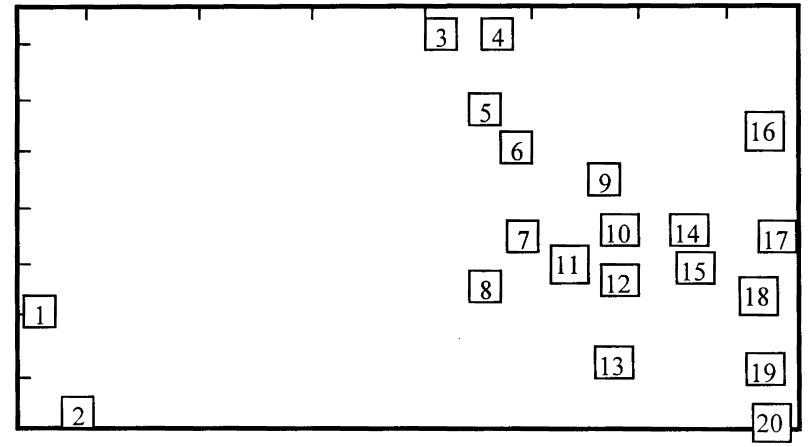

Fig. 1 The canonical analysis of physiological status: 1-Chukchi (Veseluhin, 1977); 2-Russian (Veseluhin, 1977); 3-Eskimos of Alaska (Rodahl, 1954, after Veseluhin, 1977); 4-polar explorers after 12 months of an arctic expedition (Deriapa, Riabinin, 1977); 5Eskimos of Chukot (Goudkova, 1995); 6-polar explorers after 6 months of an arctic expedition (Deriapa and Riabinin, 1977); $7-$ Khakass (Goudkova, 1998); 8-Chukchi (Goudkova, 1995); 9Shortsi (Alexeeva and Chikisheva, 1984); 10-Kamchadals (Goudkova, 1995); 11-migrants to Kamchatka (Goudkova, 1995); 12-Khakass kachintsi (Alexeeva and Chikisheva, 1984); 13-polar explorers before an arctic expedition (Deriapa and Riabinin, 1977); 14 - Turkmen before the hot season (Goudkova, 1993); 15-Khakass sagaitsi (Alexeeva and Chikisheva, 1984); 16-Nentsi (Alexeeva and Chikisheva, 1984); 17 -Karakalpaks (Goudkova, 1993); 18 -Tuvintsi (Alexeeva and Chikisheva, 1984); 19-Kazakhs (Goudkova, 1993); 20 - Turkmen in the hot season (Goudkova, 1993).

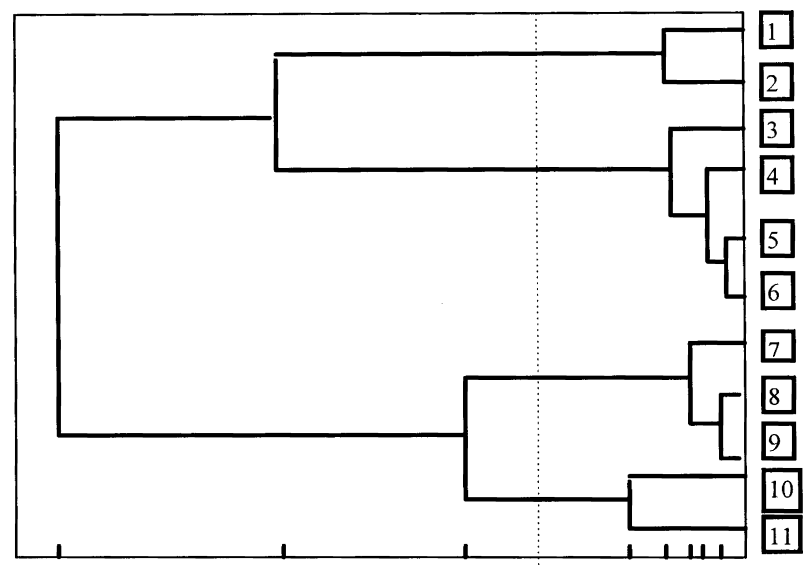

Fig. 2 The results of cluster analysis (men): 1-Karakalpaks; 2Turkmen, analysed in the hot season; 3-Turkmen, analysed before the hot season; 4-Kirgiz; 5-Tajiks; 6-Kazakhs; 7-Khakass; 8 migrants to Kamchatka; 9 -Kamchadals; 10 -Eskimos; 11 - Chukchi.

immuno-structural homeostasis as a result of insufficient adaptation to a new environment - the "stress of pioneers."

Kirgiz, Tajiks, Kazakhs and Turkmen formed a separate cluster. In spite of the fact that two populations represent the aborigines of medium altitude mountains and two represent the arid zone at sea-level the mean values of physiological blood levels are very alike. It seems that they are united in the same cluster by the ecological peculiarities (including cultural and demographic similarities) of the people of Middle Asia and Kazakhstan. From the position of the theory of physiological 


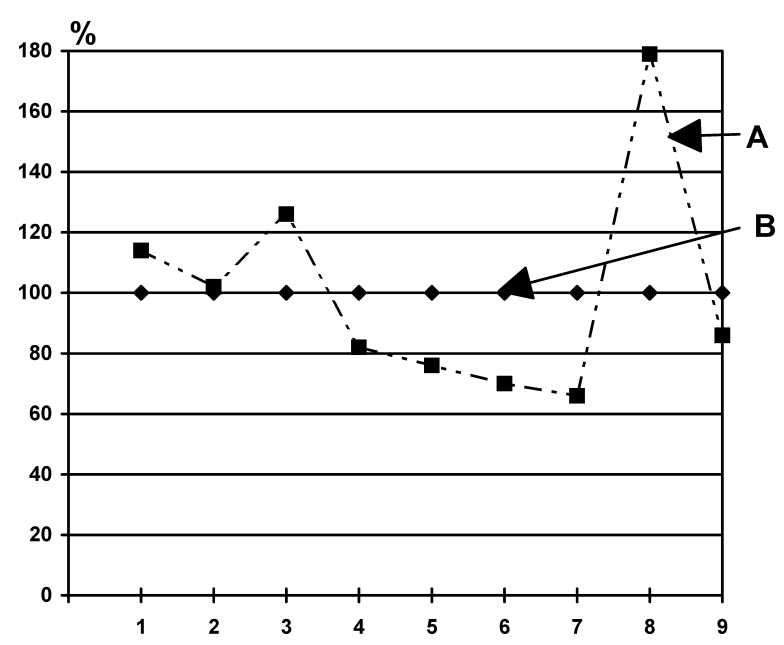

Fig. 3 The deviations of physiological blood levels (in \%) of Turkmen analysed in the hot period (A) from the Turkmen analysed before the hot period (B). 1-hemoglobin; 2-total protein; 3-albumen; 4-alpha-1-globulins; 5-alpha-2-globulins; 6-beta-globulins; 7 gamma-globulins; 8-albumen-globulin index; 9 -cholesterol.

stability (Cannon, 1932), this result may be estimated for these populations as an adaptive state (Levontin, 1981).

But it may appear that this argument is in contradiction with the fact that the populations of Turkmen and Karakalpaks investigated in the hot period, when the situation is classified as extreme, formed an independent cluster. A high air temperature causes variable physiological alterations in the organism in the desert that are aimed at stabilizing the internal temperature balance. Under the influence of the high temperature of the environment the synthesis and resynthesis of proteins is violated: the concentration of albumen, which is connected with water metabolism, becomes higher; the immunology reactions become depressed; the quantity of cholesterol becomes less; and as a compensatory reaction the level of hemoglobin becomes higher, providing a normal supply of oxygen to the tissues (Fig. 3).

The discriminate analysis indicates that the overlap of two samples out of one population is so small that only $7 \%$ of Turkmen analysed in the hot period can be diagnosed as belonging to the sample analysed before the hot period (Fig. 4). That is why two samples taken from one Turkmen population are in different clusters.

In one cluster with Turkmen, which were examined in the hot season, are Karakalpaks, which were examined also in the hot season but at the time of collecting cotton. When this is done, magnesium chlorate is employed and the contact with this defoliant lowers the hemoglobin levels, the protein levels are also changed and the activity of the alkaline phosphates decreases. As a result of the latter, the cholesterol levels reduce. The physiological status of Karakalpaks formed by the time of examination under the integral influence of extreme climatic and negative manmade factors is the reaction of a population in a more unfavourable ecological situation for health than is the case of Turkmen. For example, the high mean

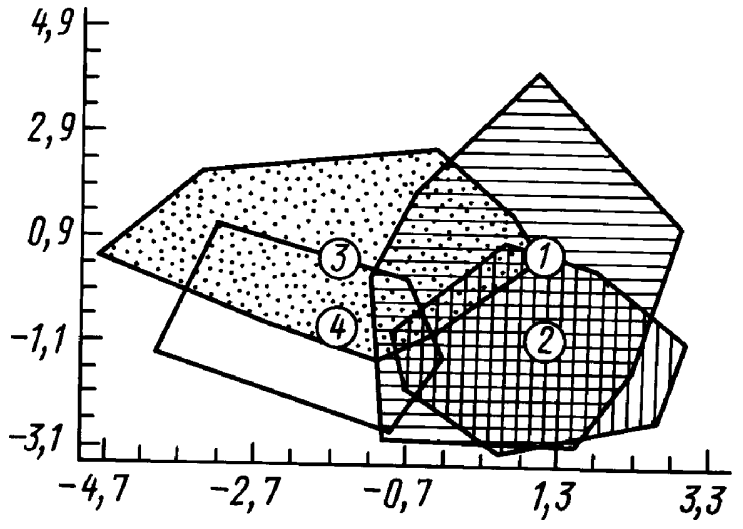

Fig. 4 The discriminant analysis of physiological status of Turkmen samples analysed in the hot season (1) and before the hot season (4), Karakalpaks analysed in the hot season (2), Kazakhs just after the hot season (3). The numbers mark the discriminant centroids of samples.

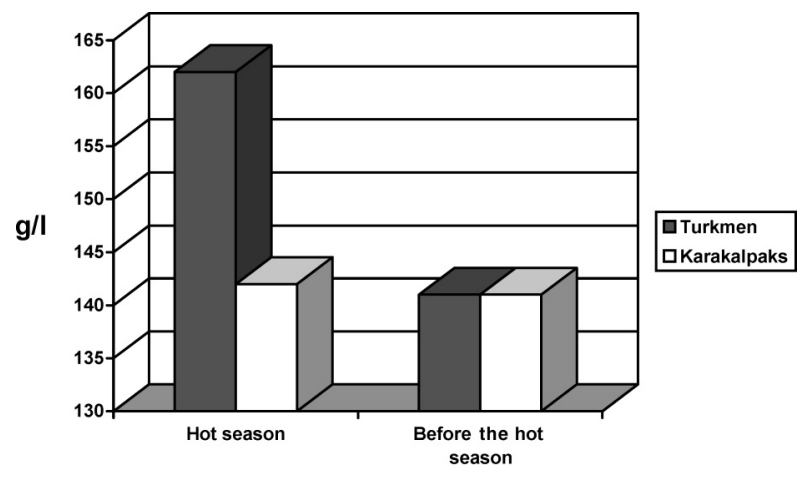

Fig. 5 The mean hemoglobin levels in Turkmen and Karakalpaks.

hemoglobin level (in the hot period), characteristic for desert inhabitants, was low among Karakalpaks. Repeated examination of hemoglobin levels before the hot season confirmed our assumption that before the defoliation the mean was equal with the Turkmen (Fig. 5).

The aborigines of Chukot, Eskimos and the Coast Chukchi formed an independent cluster. The climatic and geographical nature of the arctic zone includes a broad spectrum of extreme ecological factors and it is considered that the main factor is the radiation condition. In the variety of human reactions to the environmental influences of high latitudes, the main role is played by the physiological systems, which are connected with metabolic and energy processes. The union of two aboriginal samples of Chukot on a higher hierarchical level in comparison with other groups is a result of marked differences in their physiological status.

The difference between means in all analysed characters of Eskimos and Chukchi is statistically significant (Goudkova, 1995). This result is to some extent unexpected, because both populations are in the same ecological environment. The highest difference is in cholesterol levels: the Eskimos have the highest mean on the intragroup scale (Table 1).

The studies of the last years have shown that high 
Table 1 The means of cholesterol levels (in mmol/l) and the averaged values of variability (\%)

\begin{tabular}{l|c|c|l|l}
\hline \multicolumn{3}{c}{$\begin{array}{c}\text { The means of } \\
\text { cholesterol levels } \\
\text { (in mmol/1) }\end{array}$} & \multicolumn{1}{c}{$\begin{array}{c}\text { The averaged values of } \\
\text { variability for all } \\
\text { physiological variables } \\
\text { (Men) }\end{array}$} \\
\multicolumn{1}{c|}{ Groups } & Men & Women & \multicolumn{1}{c}{ Groups } & $\begin{array}{l}\text { The mean } \\
\text { variation } \\
\text { coefficient }\end{array}$ \\
\hline Karakalpaks & 3.63 & 3.69 & Karakalpaks & 20.0 \\
Turkmen 1 & 4.05 & 3.86 & Turkmen 1 & 21.0 \\
Turkmen 2 & 4.53 & & Turkmen 2 & 14.9 \\
Kazakhs & 4.34 & 4.11 & Kazakhs & 19.8 \\
Kirgiz & 4.37 & & Kirgiz & 16.7 \\
Tajiks & 4.61 & & Tajiks & 15.0 \\
Khakass & 5.84 & 5.82 & Khakass & 15.8 \\
Migrants & 4.71 & 4.44 & Migrants & 15.5 \\
Kamchadals & 4.50 & 4.42 & Kamchadals & 16.1 \\
Chukchi & 5.57 & 5.47 & Chukchi & 19.8 \\
Eskimo & 6.61 & 6.55 & Eskimo & 21.3 \\
\hline
\end{tabular}

(Turkmen 1 -the hot season; Turkmen 2-before the hot season)

cholesterol levels and low blood pressure are not historically dependent phenomena. The cholesterol levels and the percentage of hypercholesterolemia in Eskimos depends on the process of "food acculturation". The main difference in diet lies in the lowering of the habitual fat and protein intake and an unusually large quantity of carbohydrates, which can violate the lipid metabolism of the aboriginal people. The increase of cholesterol levels in north aborigines is also connected with regular alcohol intake (Avksentuk et al., 1995), a decline in physical activity; emotional stress provoked by urbanization and so on.

Many authors think that hypercholesterolemia often is an answer to external influences and changes in the internal situation of an organism. The stereotype character of these reactions is the evidence of their biological importance. At the beginning of stress, the violation of lipid metabolism is adaptive, but when the strength of stress rises, the changes in lipid metabolism become pathological and cause violation of the physiological homeostasis. The dynamics of the total cholesterol level as an integral indicator of the lipid metabolism gives us an opportunity to judge the adaptation reserve (Belov et al., 1988). This physiological character has the most taxonomical value also in intragroup analysis.

The organism has a definite answer to the changes in the environment, which is acquired during long previous evolution in a variable environment (Shmalgauzen, 1968). As was said in the Introduction, the population response to different conditions of the environment is the rise of phenotypic variability. But under the influence of extreme factors the levels of physiological characters of a person may change differently. The levels of some characters individually (Fig. 6) deviate in a direction not typical for the rest of the sample (Fig. $3)$. The degree of the reaction expression is very variable
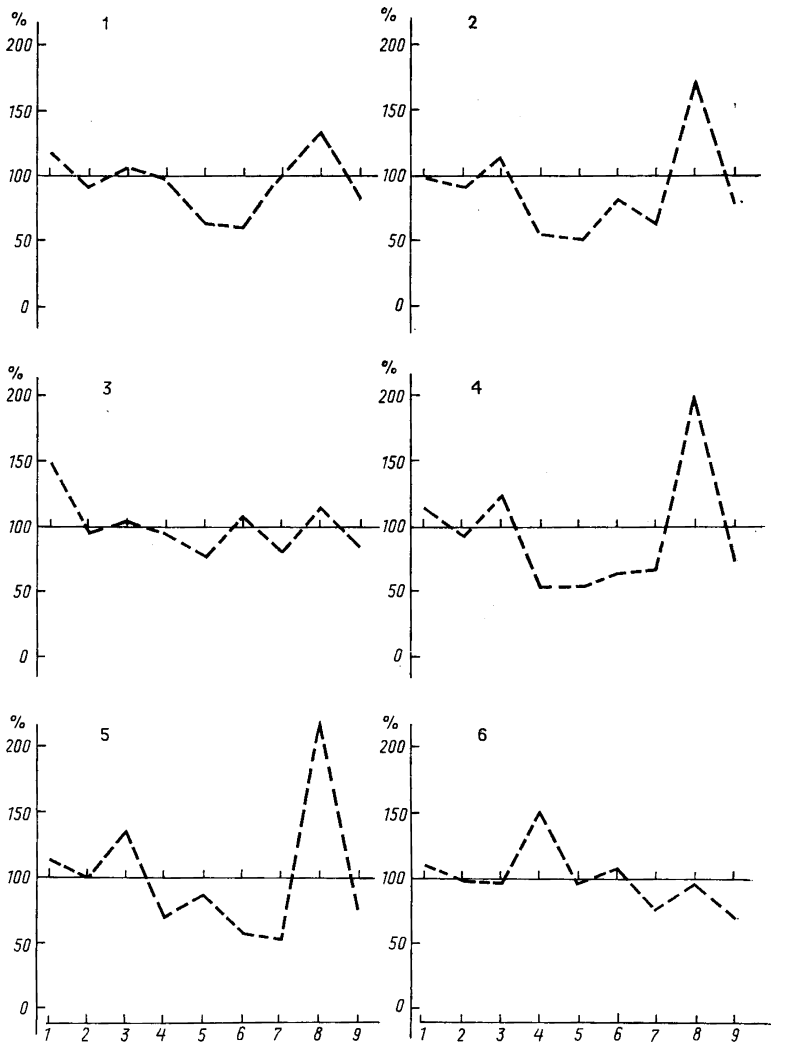

Fig. 6 The physiological blood levels in six Turkmen analysed before the hot season (horizontal line) and in the hot season. For designation see Fig. 3.

between different persons. Not one organism is perfectly adapted and in different ways and to a different degree does not correspond to the environment (Pianka, 1981).

The integrative influence of the environmental complex of factors on the population depends on the characters of every person and the existing differences between them. The adaptation to the environment of an individual, not groups or genes, culture or society (Foley, 1990), leads to a situation when under extreme conditions homeostatic disorder in every person destabilizes the equilibrium in the populationenvironment system. In a changing environment the population must have a sufficient hereditary reserve of variability to generate the needed adaptive changes (Levontin, 1981). That is why, when discussing the results of negative influences of different factors on the population, the mean values of physiological characters appear insufficient from the information point of view. The reaction of a population to unfavourable changes of the environment is also expressed in the increase of intergroup variability (Table 1) and the extension of the variability curve (Figs. 7 and 8).

For the estimation of the variability it is not sufficient to analyse the variation coefficients of separate physiological traits, because the variety of high and low variation coefficients does not have a general tendency. The estimation of overall variability may be estimated by simple "adding" and 


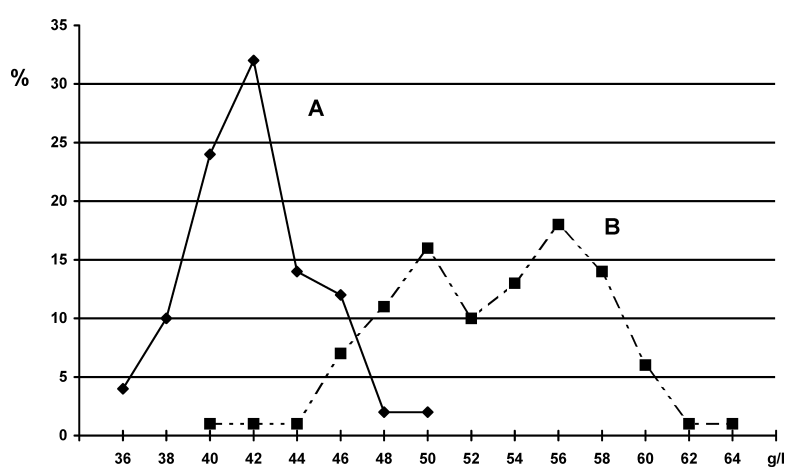

Fig. 7 The empirical distribution curves of albumen levels in Turkmen before the hot season (A) and in the hot season (B).

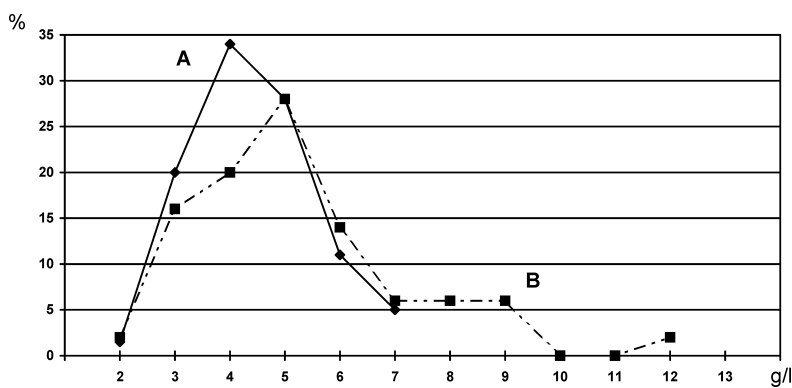

Fig. 8 The empirical distribution curves alpha-2-globulins in Chukchi (A) and Eskimo (B)

"dividing" (Table 1). But for an integral picture it is more correct to use the generalized dispersion and variation coefficient to characterize the composite system of physiological traits (Deriabin, 1982; Zhivotovsky, 1991). For example, the generalized dispersion of physiological characters in Turkmen analysed during the hot period was thirty times higher than before the hot season. The Eskimos have a substantially higher (seven times) generalized dispersion than Chukchi. The estimates of generalized variability of population physiological status can be successfully applied to when we are considering ecological problems. They not only mark the violation of the population physiological status, but also indicate the changes in the environment.

For the estimation of population physiological status dependent on the environment, correlation and factor analyses are very informative. When the correlation matrix is analysed from the point of view of the ecological peculiarities of every population, it was found that the relative number of reliable correlations grows in the populations that are in an extreme environment and the most connected traits are those which show maximum reactivity (Goudkova, 1998). The canonical correlation also grows in populations in extreme conditions (Table 2). This phenomenon, as was shown by the studies on the theory of systems, leads to the disintegration of a system with many levels (Mesarovich, 1971), which in our case marks a violation of the physiological homeostasis of populations.

The results of factor analysis of the physiological status are in Table 3. There are four main factors. They give a description
Table 2 The canonical correlation coefficients between physiological and morphological traits

\begin{tabular}{l|c|c}
\hline \multicolumn{1}{c|}{ Group } & Men & Women \\
\hline Temperate zone & $0.546(p=0.006)$ & $0.523(p=0.001)$ \\
Arid zone & $0.597(p=0.001)$ & $0.600(p=0.008)$ \\
Arctic zone & $0.792(p=0.001)$ & $0.720(p=0.001)$ \\
\hline
\end{tabular}

Table 3 The results of factor analysis of blood physiological levels (men of the temperate zone)

\begin{tabular}{l|r|r|r|r}
\hline \multirow{2}{*}{ Trait } & \multicolumn{4}{c}{ Factor } \\
\cline { 2 - 5 } & 1 & 2 & 3 & 4 \\
\hline Hemoglobin & 0.006 & 0.429 & $\mathbf{0 . 6 2 4}$ & 0.500 \\
Albumen & $-\mathbf{0 . 6 2 5}$ & $\mathbf{0 . 5 8 9}$ & 0.062 & 0.041 \\
Globulins & & - & & \\
alpha-1 & $\mathbf{0 . 6 3 4}$ & 0.017 & 0.205 & -0.160 \\
alpha-2 & $\mathbf{0 . 6 3 2}$ & 0.124 & 0.173 & -0.427 \\
beta & $\mathbf{0 . 7 0 1}$ & 0.254 & -0.320 & 0.178 \\
gamma & $\mathbf{0 . 5 1 5}$ & -0.159 & -0.218 & $\mathbf{0 . 6 6 6}$ \\
Cholesterol & 0.183 & $\mathbf{0 . 7 6 4}$ & -0.485 & -0.140 \\
Glucose & 0.382 & 0.164 & $\mathbf{0 . 5 7 9}$ & -0.126 \\
Latent roots & 2.129 & 1.248 & 1.187 & 0.971 \\
The explained part of & 26.6 & 15.6 & 14.8 & 12.1 \\
$\quad$ dispersion (\%) & & & & \\
Total explained & & 69.1 & & \\
$\quad$ variation (\%) & & & & \\
\hline
\end{tabular}

of the systemic covariability of physiological variables, which are determined by many internal and external processes taking place at the molecular, organism and population levels and on which the population physiological homeostasis depends. They are as follows: 1 -factor of integrative regulation, 2 -factor of nutrition, 3-energy factor, 4-factor of the immunity. All matrixes (for arid, arctic and temperate zones) in general are very much alike, but for the populations in an extreme environment, they have certain peculiarities (Goudkova, 2000).

Different methods of studying the variability of the physiological structure of populations gives us an opportunity to establish the difference or similarity between them in their physiological status from the point of view of ecological peculiarities, adaptation and disadaptation. Temporal unfavourable changes in the environment (for example, the climate periodicity of the arid zone) bring about changes of the population physiological status. But this process is reversible and after the change of the season, homeostasis is reestablished and the population returns to the usual state of adaptability.

A good test of the limits of human adaptive ability is the quick changes in stress that are connected with modern society (Baker, 1983), which took place in Chukot. The changing ecological reality-abrupt changes in the way of life in constantly extreme climatic and geographical conditionsaffected both Chukot populations, but the reaction of their physiological structure was different. The violation of physiological homeostasis and a number of medical and 
sociological characteristics (Goudkova, 1995) indicate a lowering of the Eskimos' adaptivness to the environment.

To the extreme Arctic environment, the Eskimos adapted for several thousand years. In modern conditions the aborigines of the North are forced to adapt to a new heterogeneous environment, which is quite different from that in which most of their natural history took place.

Keeping in mind the R. Fisher model of adaptation to an undirected worsening of the environment (Fisher, 1930, cit. after Pianka, 1981), one can propose that populations more specialized and with restricted homeostatic possibilities cannot endure substantial changes in the environment. Evidently the Eskimos are a highly specialized group the adaptive possibilities of which have expired. That is why we can conclude that the Eskimo population is in a state of disadaptation. For the Coast Chukchi, as a "younger" population comparable with Eskimos, the prognosis is more optimistic. Thus, the variability of the population physiological homeostasis reflects changes in the environment. That is why the population physiological homeostasis stability may be looked at in the context of a "stable society" (Meadows et al., 1994) as a deposit of population viability and its harmony with the inviolable environment.

\section{References}

Alexeeva TI, Chikisheva TA (1984) A comparative study of intragroup variability of some physiological traits in Tuvince. Anthropoecological studies in Tuva. Nauka, Moscow, 158-184 [In Russian]

Avksentuk AV, Kurilovich SA, Nomokonova NU (1995) The peculiarities of alcoholmetabolic enzymes of aborigine Chukot populations from the findings of molecular-genetical investigations. Health problems of the Extreme North populations in new economical conditions. Novosibirsk, 7-10 [In Russian]

Baker PT (1983) The adaptive limits of human populations. Man 19: 1-14

Belov VA, Zaletov SU, Gerasimov GM, Derbilova AV (1988) General blood cholesterol as an integral characteristic of the adaptation reserve. Clinical diagnosis: status, possibilities, perspectives. Moscow, 129-130 [In Russian]

Cannon WB (1932) The wisdom of the body. Norton, New York, 19-137

Deriabin VE (1983) Multivariate biometrics for anthropologists. Moscow, 22-35 [In Russian]

Deriapa NR, Riabinin IF (1977) Human adaptation in the polar zones of the Earth. Medicina, Leningrad, 190-237 [In Russian]
Foley R (1990) Another unique species. Mir, Moscow, 70-94 [In Russian]

Gorizontov PD (1981) Homeostasis, its mechanism and meaning. Homeostasis. Moscow, 5-27 [In Russian]

Goudkova LK (1993) The stability and variability of some physiological blood characteristics in the populations of Middle Asia and Kazakhstan. Voprosi anthropologii 87: 16-31 [In Russian]

Goudkova LK (1995) Physiological blood characteristics and the state of adaptation in the populations of North-East Asia. Voprosi anthropologii 88: 26-37 [In Russian]

Goudkova LK (1998) Physiological homeostasis in human populations (the problems of adaptation and ecology). Voprosi anthropologii 89: 3-16 [In Russian]

Goudkova LK (2000) Use of factor analysis in population physiology. Voprosi anthropologii 90: 115-126 [In Russian]

Goudkova LK (2005) Human population physiology: history, conceptions and definitions, meaning. Voprosi anthropologii 92: 56-74 [In Russian]

Levontin PC (1981) Adaptation. A Scientific Am. Book Evolution: 241-264 [In Russian]

Meadows DN, Meadows DL, Randers J (1994) Beyond the limits. Progress, Moscow, 304 [In Russian]

Mesarovich M (1988) Systems theory and biology. The point of view of a theoretician. Theory of systems and biology. Mir, Moscow, 90-128 [In Russian]

Pianka ER (1981) Evolutionary ecology. Mir, Moscow, 85-109 [In Russian]

Sapunov VB (1990) The interdependence of genetic and physiological mechanisms in adaptation on the population level. (Doctoral Thesis). Krasnoiarsk [In Russian]

Shmalgauzen II (1968) Factors of evolution. Nauka, Moscow, 266-346 [In Russian]

Solbrig OT, Solbrig DJ (1982) Introduction to population biology and evolution. Mir, Moscow, 327-331 [In Russian]

Veseluhin RV (1977) Physiological characteristic of the arctic and continental zones of the North-East Asia aborigine populations. Voprosi anthropologii 55: 36-53 [In Russian]

Zhivotovsky LA (1991) Population biometrics. Nauka, Moscow, 236-243 [In Russian]

Received: March 20, 2005

Accepted: March 27, 2005

Correspondence to: Prof. Lioudmila K. Goudkova, Kuusinena street 6-11-870, Moscow 123308, Russia

Phone: +7-095-943-3189

Fax: + 7-095-940-3964

e-mail: godina@antropos.msu.ru 